\title{
A MODEL FOR NATURAL IMMUNITY TO ASEXUAL BLOOD STAGES OF PLASMODIUM FALCIPARUM MALARIA IN ENDEMIC AREAS
}

\author{
NICOLAS MAIRE, THOMAS SMITH,* AMANDA ROSS, SETH OWUSU-AGYEI, KLAUS DIETZ, AND
} LOUIS MOLINEAUX

Swiss Tropical Institute, Basel, Switzerland; Navrongo Health Research Centre, Navrongo, Ghana; University of Tübingen, Tübingen, Germany; World Health Organization, Geneva, Switzerland

\begin{abstract}
Most mathematical models for acquired immunity to Plasmodium falciparum consider effects of immunity on duration of infection and infectiousness, but do not consider the most evident effect of immunity, which is to reduce parasite densities. Few attempts have been made to fit such models to field data. We propose a stochastic simulation model to predict the distributions of $P$. falciparum parasite densities in endemic areas, in which acquired immunity acts by reducing parasite densities. We have fitted this model to age-specific prevalence and geometric mean densities from settings in Ghana, Nigeria, and Tanzania. The model appears to reproduce reasonably well the parasitologic patterns seen in malariologic surveys in endemic areas and is appropriate for predicting the impact of interventions such as vaccination in the context of continual exposure to $P$. falciparum.
\end{abstract}

\section{INTRODUCTION}

In areas endemic for Plasmodium falciparum malaria, many people are subjected to frequent re-infection; thus, they develop partial immunity that leads to control of parasite densities and to reduction in the frequency of clinical episodes. ${ }^{1}$ However, the level of acquired immunity does not reach a state of absolute resistance to infection. Mathematical models that capture these effects of immunity are needed to predict the potential epidemiologic impact of partially effective interventions against the parasite, such as current formulations of malaria vaccines. ${ }^{2}$

Within-host models for individual $P$. falciparum infections have considered how partial immunity affects parasite densities as an explicit consequence of differential survival of the circulating asexual parasites as the infection develops. ${ }^{3,4}$ However, models for the dynamics of $P$. falciparum in populations, including those modeling acquired immunity, ${ }^{5-7}$ have generally not considered the densities of asexual parasites. We are aware of only one population model for $P$. falciparum malaria that explicitly considered the effects of immunity on parasite densities. ${ }^{8}$ The lack of models of parasite density is surprising because effects on densities are the clearest evidence for an effect of naturally acquired immunity. The existence of such natural immunity represents some of the strongest evidence that development of an efficacious malaria vaccine is possible.

One of the major effects of immunity in most epidemiologic models of malaria ${ }^{6,7,9}$ is to reduce the duration of infections and consequently the number of infections of the vector resulting from one human infection. This quantity, following the report of Macdonald, ${ }^{10}$ is generally assumed to be proportional to infection duration, which leads to a formula that makes the basic reproductive number proportional to the infection duration. However, there is little empirical evidence on the effect of acquired immunity on duration of infection. ${ }^{11}$ Some models ${ }^{12}$ and empirical studies ${ }^{13,14}$ have even suggested that in the semi-immune host, chronic malaria infection may persist longer than in naive hosts.

We now propose a new model for natural immunity to the

\footnotetext{
* Address correspondence to Thomas Smith, Swiss Tropical Institute, Socinstrasse 57, PO Box, CH-4002, Basel, Switzerland. E-mail: nicolas.maire@unibas.ch, Thomas-A.Smith@unibas.ch
}

asexual blood stages of $P$. falciparum that specifically focuses on the effect on parasite densities, since it is undoubtedly the case that the major impact of acquired immunity is to reduce the overall parasite load in infected individuals. This model makes predictions of the age patterns of patent parasitemia and of the geometric mean parasite density. Infections in naive hosts mimic the levels of parasitemia reached by $P$. falciparum infections induced to treat neurosyphilis in the United States (Milledgeville Hospital, Milledgeville, GA and $\mathrm{Na}-$ tional Institutes of Health Laboratories, Columbia, SC from 1940 to 1963 , Figure 1). ${ }^{15}$ In previously exposed hosts, we model a reduction in the densities that depends on the host's history of infection. This simulated acquired immunity does not affect the duration of the infections although it can reduce the period for which parasite density is above the detection limits used in field malariology.

We implement the model by stochastic simulation of each individual infection in a human population using a five-day time-step, and introduce infections by a process dependent on the temporal pattern of the entomologic inoculation rate (EIR) modulated as described. ${ }^{16}$

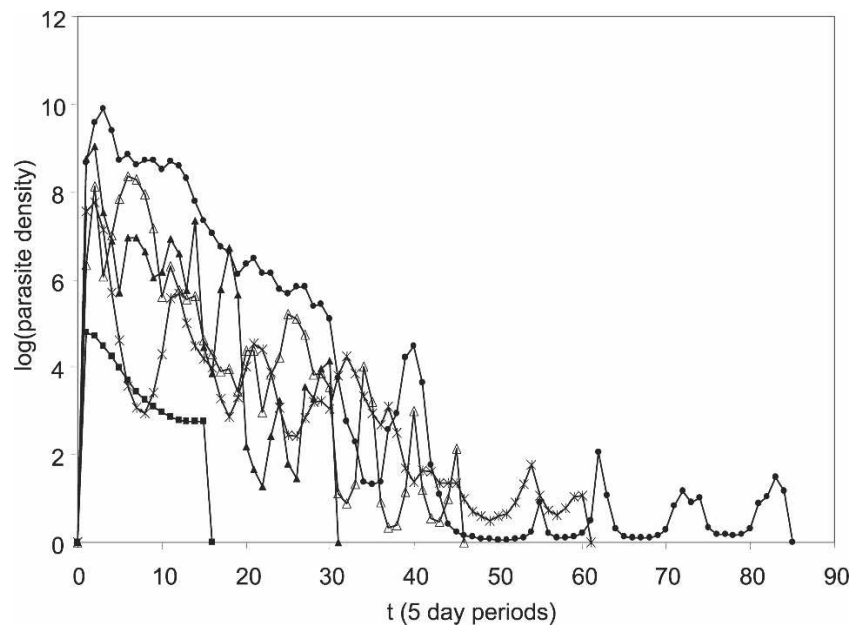

FIgURE 1. Parasite density as a function of time since infection in malaria-naive individuals. Geometric mean parasite density as a function of time since start of patency and depending on the duration of patent infection. The figure shows examples from five individual patients. 
Here, we present how the model has been fitted to prevalence, density, and multiplicity data of $P$. falciparum obtained from different epidemiologic settings in Ghana, Nigeria, and Tanzania, and show that it gives realistic predictions of ageprevalence and age-density relationships across a range of transmission intensities. This model forms one component of a comprehensive dynamic model for the transmission cycle of the $P$. falciparum parasite, and of malaria morbidity, and mortality, as well as of cost-effectiveness of control strategies.

\section{METHODS}

Epidemiologic data. The model was fitted simultaneously to six datasets of pre-intervention cross-sectional malariologic surveys from four different field studies of $P$. falciparum malaria in Africa (Tables 1 and 2) that were chosen to represent a range of transmission intensities. In addition, datasets from Saradidi, Kenya and from interventions carried out in Matsari village (Garki project), Nigeria were used to fit the model for the incidence of infection, as described in the accompanying paper. ${ }^{16}$ Data from the pre-intervention phase of the Garki project collected in Rafin Marke, Matsari, and Sugungum villages, and from Navrongo, Idete, and Namawala were used to fit the model for three distinct cross-sectional outcomes in the absence of intervention: 1) age-specific prevalence of patent parasitemia, 2) age-specific geometric mean density of parasitemia in positive individuals, and 3) age-specific multiplicity measured by polymerase chain reaction-restriction fragment length polymorphisms (PCR-RFLP) of the merozoite surface protein $2^{16}$ gene (data from Navrongo only).

In all of these field studies, entomologic surveys had been carried out to determine the annual cycle of the inoculation rate (Figure 2 and Table 1) and these entomologic data were used as input to the simulation model. In all sites except Idete, the health system at the time of the surveys treated only a small proportion of the clinical malaria episodes; in the Idete study, we calculate from the published results ${ }^{17,18}$ that the village dispensary treated approximately $64 \%$ of clinical malaria attacks and our simulations assume this coverage of effective treatment. Parasite densities in the Garki dataset were recorded by scanning a predetermined number of microscope fields on the thick blood film and recording how many had one or more asexual parasites visible. We converted these values to numbers of parasites/microliter by assuming a Poisson distribution for the number of parasites per field and a blood volume of $0.5 \mathrm{~mm}^{3}$ per 200 fields. ${ }^{9}$ In the other field studies, parasites were counted against leukocytes and converted to nominal parasites/microliter assuming the usual (though biased) standard of 8,000 leukocytes/microliter. ${ }^{19}$ The biases in density estimates resulting from these different

TABLE 1

Datasets included in the fitting of the model to field data*

\begin{tabular}{|c|c|c|c|c|}
\hline Site/reference & Description of the data & Purpose of inclusion & EIR & Quantities fitted \\
\hline $\begin{array}{r}\text { Rafin-Marke, Garki, Nigeria } \\
\text { (pre-intervention phase) } \ddagger^{9}\end{array}$ & $\begin{array}{l}8 \text { cross-sectional surveys of entire } \\
\text { village population, at } 10 \text {-week } \\
\text { intervals (total } 2,593 \text { blood slides) }\end{array}$ & $\begin{array}{l}\text { Age-prevalence and density at } \\
\text { lowest EIR in the Garki } \\
\text { project }\end{array}$ & 9 & $\begin{array}{l}\text { Age- and season-specific } \\
\text { parasite prevalence, } \dagger \text { age- } \\
\text { specific parasite density } \dagger\end{array}$ \\
\hline $\begin{array}{l}\text { Matsari, Garki, Nigeria } \\
\quad \text { (pre-intervention phase) } \ddagger^{9}\end{array}$ & $\begin{array}{l}8 \text { cross-sectional surveys of entire } \\
\text { village population, at } 10 \text {-week } \\
\text { intervals (total 2,963 slides) }\end{array}$ & $\begin{array}{l}\text { Age-prevalence and density at } \\
\text { circa the median EIR for the } \\
\text { Garki project }\end{array}$ & 9 & $\begin{array}{l}\text { Age- and season-specific } \\
\text { parasite prevalence, } \dagger \text { age- } \\
\text { specific parasite density } \dagger\end{array}$ \\
\hline $\begin{array}{l}\text { Sugungum, Garki, Nigeria } \\
\quad \text { (pre-intervention phase) } \ddagger^{9}\end{array}$ & $\begin{array}{l}8 \text { cross-sectional surveys of entire } \\
\text { village population, at } 10 \text {-week } \\
\text { intervals (total } 4,487 \text { blood slides) }\end{array}$ & $\begin{array}{l}\text { Age-prevalence and density at } \\
\text { highest EIR in the Garki } \\
\text { project }\end{array}$ & 9 & $\begin{array}{l}\text { Age- and season-specific } \\
\text { parasite prevalence, } \dagger \text { age- } \\
\text { specific parasite density } \dagger\end{array}$ \\
\hline Saradidi, Kenya ${ }^{25}$ & $\begin{array}{l}21 \text { cohorts each of approximately } \\
50 \text { children between } 6 \text { months } \\
\text { and } 6 \text { years of age whose para- } \\
\text { sites were cleared and who were } \\
\text { then followed-up with } 2 \text { weekly } \\
\text { surveys. }\end{array}$ & $\begin{array}{l}\text { Incidence of infection in relation } \\
\text { to EIR }\end{array}$ & 21 & $\begin{array}{l}\text { Incidence risk of infection } \\
\text { over } 2 \text {-week periods }\end{array}$ \\
\hline $\begin{array}{l}\text { Matsari, Garki, Nigeria } \\
\quad\left(\text { intervention phase) }{ }^{9}\right.\end{array}$ & $\begin{array}{l}8 \text { cross-sectional surveys of entire } \\
\text { village population, at } 10 \text {-week } \\
\text { intervals. Mass treatment with } \\
\text { sulfamine-pyrimethamine } 10- \\
\text { weeks prior to each survey (total } \\
2,663 \text { blood slides) }\end{array}$ & $\begin{array}{l}\text { Incidence of infection allowing } \\
\text { for patency of infections }\end{array}$ & 9 & $\begin{array}{l}\text { Age- and season-specific } \\
\text { incidence risk of patent } \\
\text { infection } \dagger\end{array}$ \\
\hline Namawala, Tanzania ${ }^{24}$ & $\begin{array}{l}12 \text { cross-sectional surveys of an age- } \\
\text { stratified sample, at } 2 \text {-month } \\
\text { intervals (total 3,901 blood slides) }\end{array}$ & $\begin{array}{l}\text { Age-prevalence and density at } \\
\text { very high EIR }\end{array}$ & 24 & $\begin{array}{l}\text { Age- and season-specific } \\
\text { parasite prevalence, } \dagger \text { age- } \\
\text { specific parasite density } \dagger\end{array}$ \\
\hline Idete, Tanzania ${ }^{17,45}$ & $\begin{array}{l}\text { Surveillance of a rolling cohort of } \\
\text { infants }(1,382 \text { blood slides over } \\
16 \text { months). Also } 1 \text { cross-sec- } \\
\text { tional survey of } 312 \text { children } 1-5 \\
\text { years old } \$\end{array}$ & Effects of maternal immunity & 23 & $\begin{array}{l}\text { Age- and season-specific } \\
\text { parasite prevalence, } \dagger \text { age- } \\
\text { specific parasite density } \dagger\end{array}$ \\
\hline Navrongo, Ghana ${ }^{31}$ & $\begin{array}{l}6 \text { cross-sectional surveys of an age- } \\
\text { stratified sample, at } 2 \text {-month } \\
\text { intervals (total } 522 \text { slides/DNA } \\
\text { samples) }\end{array}$ & $\begin{array}{l}\text { Age-multiplicity and seasonality } \\
\text { of multiplicity }\end{array}$ & II & $\begin{array}{l}\text { Age-specific parasite preva- } \\
\text { lence and density,* age- } \\
\text { specific multiplicity of msp-2, } \\
\text { by PCR-RFLP }\end{array}$ \\
\hline
\end{tabular}


TABLE 2

Overall data summary and log likelihoods for each site*

\begin{tabular}{|c|c|c|c|c|c|c|}
\hline \multicolumn{4}{|c|}{ Data summary } & \multicolumn{3}{|c|}{ Loss functions for best fitting model } \\
\hline & $\underset{\text { EIR }}{\text { Annual }}$ & $\begin{array}{c}\% \\
\text { Prevalence } \dagger\end{array}$ & $\begin{array}{c}\text { Geometric } \\
\text { mean density } \\
\text { (parasites/ } / \mathrm{L}) \ddagger\end{array}$ & $\begin{array}{l}\text { Log likelihood } \\
\text { for prevalence }\end{array}$ & $\begin{array}{l}\text { Log likelihood } \\
\text { for geometric } \\
\text { mean density }\end{array}$ & $\begin{array}{l}\text { Log likelihood } \\
\text { for multiplicity }\end{array}$ \\
\hline Sugungum & 132 & 48.3 & 22.4 & $-2,830.4$ & -116.6 & - \\
\hline Rafin Marke & 18 & 44.2 & 17.8 & $-1,722.4$ & -131.2 & - \\
\hline Matsari (pre-intervention phase) & 68 & 41.6 & 18.4 & $-1,895.9$ & -122.8 & - \\
\hline Matsari (intervention phase) & $5.5 \S$ & 8.5 & - & -782.4 & - & - \\
\hline IdeteII & 584 & 59.6 & $3,146.8$ & $-1,124.3$ & -132.7 & \\
\hline Navrongo & 405 & 54.8 & 336.1 & -341.7 & -116.1 & -276.8 \\
\hline Namawala & 329 & 77.3 & $1,111.3$ & $-2,007.7$ & -92.1 & - \\
\hline
\end{tabular}

$*$ EIR $=$ entomologic inoculation rate

$\dagger$ Overall proportion of slides positive for Plasmodium falciparum.

$\ddagger$ Geometric mean of recorded parasite densities on positive slides. The substantial difference between the Garki study and other datasets partly reflects different parasitologic methods and is allowed for by the bias adjustment in the models.

$\S$ Arithmetic mean of estimated daily inoculation rate for the whole intervention period based on re-analysis of the original data.

If Average parasite densities in Idete are much higher because this dataset comprises predominantly data for very young children. In all cases the models were fitted to the age group- and survey-specific data, not to the overall means. The data from Saradidi and the fit to those data are described in an accompanying paper. ${ }^{16}$

techniques was accounted for by multiplying the observed parasite densities with constant values estimated for Garki $\left(v_{0}\right)$ and non-Garki $\left(v_{1}\right)$ field studies to rescale them to the values in the malariatherapy patients (determined using the methods of Earle and Perez ${ }^{20}$ ).

Hypothesized set of processes controlling asexual parasitemia. For every individual in the simulated population each discrete infection is characterized by simulated duration and densities at each five-day time point. The host acquires immunity as a function of exposure and this in turn modifies the density of subsequent infections.

Durations of infection. We treat each infection as monoclonal, but note that the infection process allows multiple infections during the same time interval. Each new infection $j$, initiated in individual $i$ at time $t_{0}$, is assigned a duration, i.e., $t_{\max }(i, j)$. The duration is defined as the time interval between the first and last days of patent parasitemia that would be observed in the absence of adjustments to allow for host immunity. The present model does not allow the immune status of the host to affect the simulated period for which the infection persists, although the immune status does affect the duration of patent infection by modifying the proportion of time points for which the parasites are at detectable densities.

The simulated infection starts after the pre-erythrocytic latent period $l_{p}$, set to three time intervals, i.e., 15 days that correspond to the hepatic stage of the life cycle plus the prepatent blood stage infection. We use the index $\tau$ to denote the time since the start of patent infection, i.e., $\tau=1$ corresponds to the first five-day interval when the infection becomes patent, three time units after the infection event.

The duration of infection is randomly sampled from a distribution equivalent to that of malariatherapy patients treated in Milledgeville, Georgia. ${ }^{15}$ Infections in patients from this hospital persisted for longer than those in South Carolina, and this appears to reflect more conservative policies (more treatment) in the latter hospital. We therefore assume that the Georgia infections were more similar to untreated natural infections in a typical malaria-endemic setting of Africa. The period of follow-up after the last positive slide also varied. Thus, confidence that an infection was spontaneously cleared also varied. We considered only patients who did not receive any anti-malarial treatment on their last day of positivity, and for each of these determined $t_{1}(i)$, the duration of follow-up after the last recorded day of positivity. For a series of cutoffs, $t_{1}^{*}$, we considered that subset of patients for whom $t_{1}(i)>t_{1} *$, and calculated the mean of the logarithm of the observed durations for those patients who were included. We surmise that the subset giving the highest value of this mean best approximates the behavior of untreated natural infections. The maximum value of this mean was 5.13 (corresponding to a geometric mean of 169.0 days), which was computed from a subset comprising those 47 patients for whom $t_{1}(i)>2$ months. In this set of patients, the standard deviation of the natural $\log$ of the duration was 0.80 , and the distribution of durations in this subset of patients was approximately log normal. In the simulations we therefore sampled the durations of untreated infections using

$$
\ln \left(\tau_{\max }(i, j)\right) \sim \operatorname{Normal}(5.13,0.80)
$$

Expected densities of single infections. At each time point, $\tau=0,1, \ldots \tau_{\max }(i, j)$, the density, $y(i, j, \tau)$, of the infection $j$ in host $i$, is set by first determining the expected log density, $E\left(\ln \left(y_{0}(i, j, \tau)\right)\right)$, that would apply in the absence of previous exposure. To determine $E\left(\ln \left(y_{0}(i, j, \tau)\right)\right)$, we first determine the mean logarithm of the densities of malariatherapy patients in the Georgia hospital specific for the age of the infection $(\tau)$ and for the pre-defined duration $\left(t_{\max }\right)$. The distributions of densities for the Georgia patients were summarized by grouping the data into five-day categories according to the time since the first day of patent asexual parasitemia and according to durations, and then using moving average smoothing of the logarithmically transformed densities to obtain a function, $y_{G}\left(\tau, t_{\max }\right)$ (Figure 1$)$, which is a simple description of average densities experienced in the course of a single malaria infection of a previously naive host.

The actual densities experienced by different malaria therapy patients varied widely even when the inocula were of the same strain, and were not greatly affected by the size of the inoculum. ${ }^{21}$ In our model this between-host variation is captured by assigning to each individual $i$ in the simulated population a value, $(d(i))$, drawn from a log-normal distribution (geometric mean 1 , variance $\sigma_{i}^{2}$ ), which multiplies the densities so that

$$
E\left(\ln \left(y_{0}(i, j, \tau)\right)\right)=\ln d(i)+\ln \left(y_{G}\left(\tau, t_{\max }\right)\right)
$$

This empirically determined function provides a description of single infections that thus captures the effects of blood 

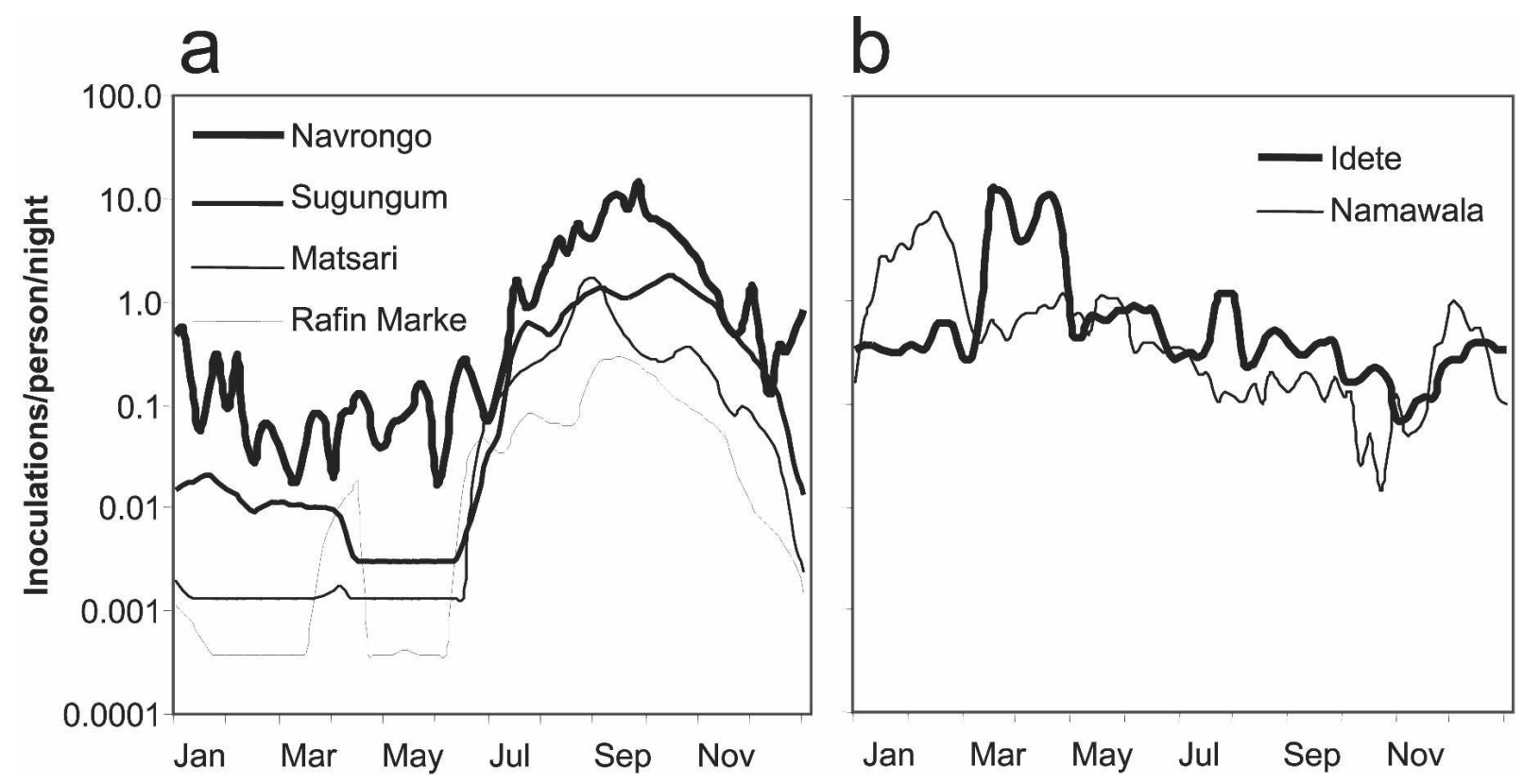

FIGURE 2. Entomologic input data Annual cycle of the entomologic inoculation rate. a, West African sites. b, East African sites.

stage immunity that the infection stimulates against itself. This includes effects of innate immunity, antigenic variation, and variant independent anti-merozoite immunity. ${ }^{3}$

Adjustments are then applied to $E\left(\ln \left(y_{0}(i, j, \tau)\right)\right)$ to allow for immunity acquired as a result of exposure to previous infections and for co-infection in hosts with multiple infections. These determine the adjusted expected value, $E(\ln (y(i, j, \tau)))$. The simulated density $y(i, j, \tau)$ is then determined by sampling its logarithm using normal distributions centered on $E(\ln (y(i, j, \tau)))$.

Effects of acquired immunity on the expected parasite density. Acquired immunity to erythrocytic stages of the parasite is related to cumulative exposure to asexual parasites. In our model the extent of acquired immunity depends both on the diversity of the parasites to which the host has been exposed and on the cumulative density of total parasitemia. In the current version we assume no decay of this component of natural immunity in the absence of infections.

The first trigger function is the cumulative density (parasites/microliter of blood $\times$ days) of asexual parasitemia since birth up to time $t$ for individual $i$, i.e.,

$$
X_{y}(i, j, t)=\int_{t-a}^{t} Y(i, \tau) d \tau-\int_{t_{0, j}}^{t} y(i, j, \tau) d \tau
$$

where $Y(i, \tau)$ is the total parasite density of individual $i$ at time $\tau$, and $a$ is the age at time $t . X_{y}(i, j, t)$ measures the total antigenic stimulus to which the host has been exposed less the exposure due to infection $j$, which is measured by the term $\int_{t_{0, j}}^{t} y(i, j, \tau) d \tau$. The latter term must be subtracted to avoid allowing twice for acquired immunity stimulated by infection $j$ itself.

We parameterize the effect $\left(D_{y}\right)$ of this exposure on parasite densities with a Hill function of the form

$$
D_{y}=\frac{1}{1+\frac{X_{y}}{X_{y}^{*}}}
$$

where we omit the indices from $X_{y}(i, j, t)$ to simplify the notation, and where $X_{y}^{*}$ is a parameter to be estimated, which takes a real positive value, and where $D_{y}$ consequently takes a value 1 when $X_{y}=0$, and is small but positive when $X_{y}$ is large.

The second trigger function is the cumulative number of prior infections that the host has experienced, i.e.,

$$
X_{h}(i, t)=\int_{t-a}^{t} h(i, \tau) d \tau-1
$$

This term monotonically increases with the size of the repertoire of non-variant polymorphic antigens to which the host has been exposed, and therefore provides a measure of the antigenic stimulus that they provide. We assume that the effect of this on parasite densities can be measured by a further, one-parameter sigmoidal function where the further parameter $X_{h}^{*}$ is constrained to take a positive value, i.e.,

$$
D_{h}=\frac{1}{1+\frac{X_{h}}{X_{h}^{*}}} .
$$

The third trigger function is the age of the host $a$, which is inversely related to the extent of maternally derived protection. We treat the level of maternal protection as independent of maternal exposure. In case of a low transmission level, few infants will be exposed during the first few months of life, so maternal immunity is irrelevant. If transmission is frequent, then all mothers will have similar immune status. ${ }^{22}$

We parameterize the multiplication factor that models the effect of maternal immunity $D_{m}$ with a decay function that lies between 0 (maximal effect, corresponding to the status at birth), and 1 (no reduction in density), such that

$$
D_{m}=1-\alpha_{m} \exp \left(-\frac{0.693 a}{a_{m}^{*}}\right)
$$

where $a_{m}^{*}$, the half-life of the maternal immunity, and $\alpha_{m}$, the 
maternal protection at birth, are additional parameters to be estimated. We then compute the expected density for single infections as

$$
E(\ln (y(i, j, \tau)))=D_{y} D_{h} D_{m} \cdot \ln \left(y_{0}(i, j, \tau)\right)
$$

Effects of concurrent infections. When the host is infected with more than one infection at the same time, innate immunologic responses, together with other density-dependent regulatory mechanisms, lead to a reduction in the overall density to below that expected if the infections did not interact. We estimate this effect by assuming the density of each component infection to be multiplied by a term constrained to take a value between 1 (no interaction between co-infections) and $1 / M(t)$, where $M(t)$ is the total multiplicity of concurrent infections at time $t$. The value $1 / M(t)$ corresponds to reduction of the total density to that expected if there was only a single infection. To achieve this we define a further parameter $D_{x}$, constrained to be between 0 and 1 and set

$$
E(\ln (y(i, j, \tau)))=D_{y} D_{h} D_{m} \cdot \ln \left(y_{0}(i, j, \tau)\right)+\ln \left(\frac{D_{x}}{M(t)}+1-D_{x}\right)
$$

Distributions of parasite densities and determination of expected prevalence. The predicted densities for each individual in the simulated population, $y(i, j, \tau)$, are sampled around the expectations of their logarithms, using log-normal distributions. The variance of these log-normal distributions, which is estimated from the field data during the fitting process, comprises both variation between hosts, and variation within individual hosts.

Variation between hosts is quantified by the term $\sigma_{i}^{2}$, while variation within individual hosts is quantified by a term $\sigma_{y}^{2}(i, j, \tau)$, which includes an effect of the cumulative exposure of the host, and is necessary to obtain a good fit. Thus,

$$
\sigma_{y}^{2}(i, j, \tau)=\frac{\sigma_{0}^{2}}{1+\frac{X_{h}(i, t)}{X_{v}^{*}}}
$$

where the parameters $\sigma_{0}^{2}$ and $X_{v}^{*}$ need to be estimated.

It follows that the simulated densities are distributed as

$$
\ln (y(i, j, \tau)) \sim \operatorname{Normal}\left(E(\ln (y(i, j, \tau))), \sigma_{y}^{2}(i, j, \tau)\right)
$$

The total density at time $t$ in host $i$ is then the sum of the densities of the various co-infections $j$.

$$
Y(i, t)=\sum_{j} y(i, j, \tau(i, j))
$$

Entomologic inoculation rate. The input to the model is the annual cycle of the EIR. In the case of the Garki sites in Nigeria, the EIR was assessed using human night bait collections and dissections of the salivary glands of the Anopheles mosquitoes to give the sporozoite rate. A random effects logistic model was used to give season- and village-specific estimates of the sporozoite rate. The EIR values were then rescaled so the annual totals corresponded to the published values of 18, 68, and 132 for the villages of Rafin Marke, Matsari, and Sugungum, respectively 9 .

In the cases of Idete (annual EIR $=584$ ) and Namawala (annual EIR = 329) in Tanzania and Navrongo in Ghana (annual EIR = 418), the human biting rate was calculated using light trap collections, with adjustment for the relative numbers of mosquitoes caught by human landing and light traps. ${ }^{23,24}$ The sporozoite rates were estimated by using an enzyme-linked immunosorbent assay (ELISA) to test the heads and thoraces of the captured anophelines for sporozoites.

For each of the sites, which were assumed to be at equilibrium, an estimate of the daily inoculation rate was assigned to each five-day period, based on re-analysis of the original data, and averaging estimates for the same season, where data were available from multiple years. Where the observed dry season biting rate was too low for an estimate of the EIR to be made, a value was assigned equal to $1 \%$ of the average rate for the rest of the year. The inoculation rate estimates for Saradidi in Kenya, based on human landing collections and sporozoite ELISA techniques, were taken directly from the report by Beier and others. ${ }^{25}$

Implementation and fitting of the model. Since the model parameters are not identifiable from the data from single transmission settings or from single outcome measures, estimates were made by maximizing the joint likelihood for all of the quantities listed in Table 1. Each likelihood evaluation required a stochastic individual-based simulation of a human population comprising 10,000 individuals for each of the 6 datasets (corresponding to the malariologic patterns in 6 different African villages). These simulations were implemented in Fortran 95 using five-day time steps, with as input the EIR for each five-day period. Each simulation began by simulating the exposure of the population over a whole lifetime prior to the period for which the data applied (to ensure the correct values of the cumulative exposure variables at the start of the monitoring period). For this warm-up period the same average annual cycle of the EIR (estimated from the available entomologic data) was assumed to have recurred since the birth of the oldest member of the simulated population. A demographic model was used that ensured that the simulated population remained stable with the same age-distribution throughout (Appendix).

The actual surveys carried out in the six villages were simulated, and predictions thus made for the distribution of parasite densities in each age group at each survey. Simulated prevalence was defined by comparing each predicted parasite density with the limit of detection used in the actual field study. By comparing observed and simulated geometric mean densities we were able to optimize the parameters of our model for immunologic control of parasite densities. The comparison of the observed with simulated prevalence allowed us at the same time to estimate the variances of the parasite density distributions.

The likelihood was computed for each of the outcomes listed in Table 1 separately for each age group and each survey. Binomial likelihoods were used for the prevalence of patent parasitemia, and a normal likelihood for the mean log parasite density among slides positive by microscopy. For the Navrongo dataset Poisson likelihoods were calculated for the total numbers of distinct parasite infections detected by polymerase chain reaction-restriction fragment length polymorphism in the sampled individuals in each age group, and at each survey.

The overall loss function was computed as a weighted sum of the negative log likelihoods for each of these components across all age-groups, surveys, and sites (Tables 1 and 2). 
TABLE 3

Fitted values of the parameters*

\begin{tabular}{|c|c|c|c|c|}
\hline Parameter & Process quantified & Units/dimension & Equation & Point estimate $(95 \% \mathrm{CI})$ \\
\hline$Y_{h}^{*}$ & Critical value of exposure to parasites for liver stage immunity & Parasite-days $/ \mu \mathrm{L}$ & equation $9^{16}$ & $\infty$ \\
\hline$X_{*}^{*}$ & Effect of exposure on parasite density, critical value & Parasite-days $/\left(\mu \mathrm{L} \times 10^{-7}\right)$ & 3 & $3.5(2.9,4.2)$ \\
\hline$X_{h}^{*}$ & Effect of exposure on number of infections, critical value & Infections & 5 & $97.3(20.0,474.5)$ \\
\hline$\alpha_{m}$ & Maternal protection at birth & Proportion & 7 & $0.90(0.88,0.93)$ \\
\hline$a_{m}^{*}$ & Decay of maternal protection & Per year & 7 & $2.53(2.33,2.75)$ \\
\hline$D_{x}$ & Effects of concurrent co-infections on density & Dimensionless & 8 & 0 \\
\hline$\sigma_{i}^{2}$ & Variation between individuals in densities & Dimensionless & 2 & $10.2(9.7,10.7)$ \\
\hline$\sigma_{0}^{2}$ & Fixed variance component for densities & $\ln ($ density) & 10 & $0.66(0.54,0.79)$ \\
\hline$X_{v}^{*}$ & Critical value of the exposure in the model of variance & Infections & 10 & $0.92(0.82,1.03)$ \\
\hline$v_{0}$ & Bias adjustment for observed parasite densities in sites from Garki & Dimensionless & - & $4.80(4.28,5.38)$ \\
\hline$v_{1}$ & Bias adjustment for observed parasite densities in non-Garki sites & Dimensionless & - & $0.18(0.17,0.19)$ \\
\hline
\end{tabular}

Each age-specific prevalence or multiplicity assessment was given unit weight, but we weighted the log likelihoods for parasite densities by a factor of 10 (so that the prevalence and density had roughly equal weight in the final loss function). We also included in the fitting process simulation of the infection process during the intervention phase in Matsari village $^{16}$ (model B). The log-likelihood for the prevalence data from this simulation was also subtracted from the overall loss function (Table 2). The goodness of fit of the model to the data was assessed graphically.

Simulated annealing ${ }^{26,27}$ was used to identify the values of the parameters that minimized the loss function (Table 3). Approximate confidence intervals were obtained by estimating the Fisher information for the parameters. This was done a
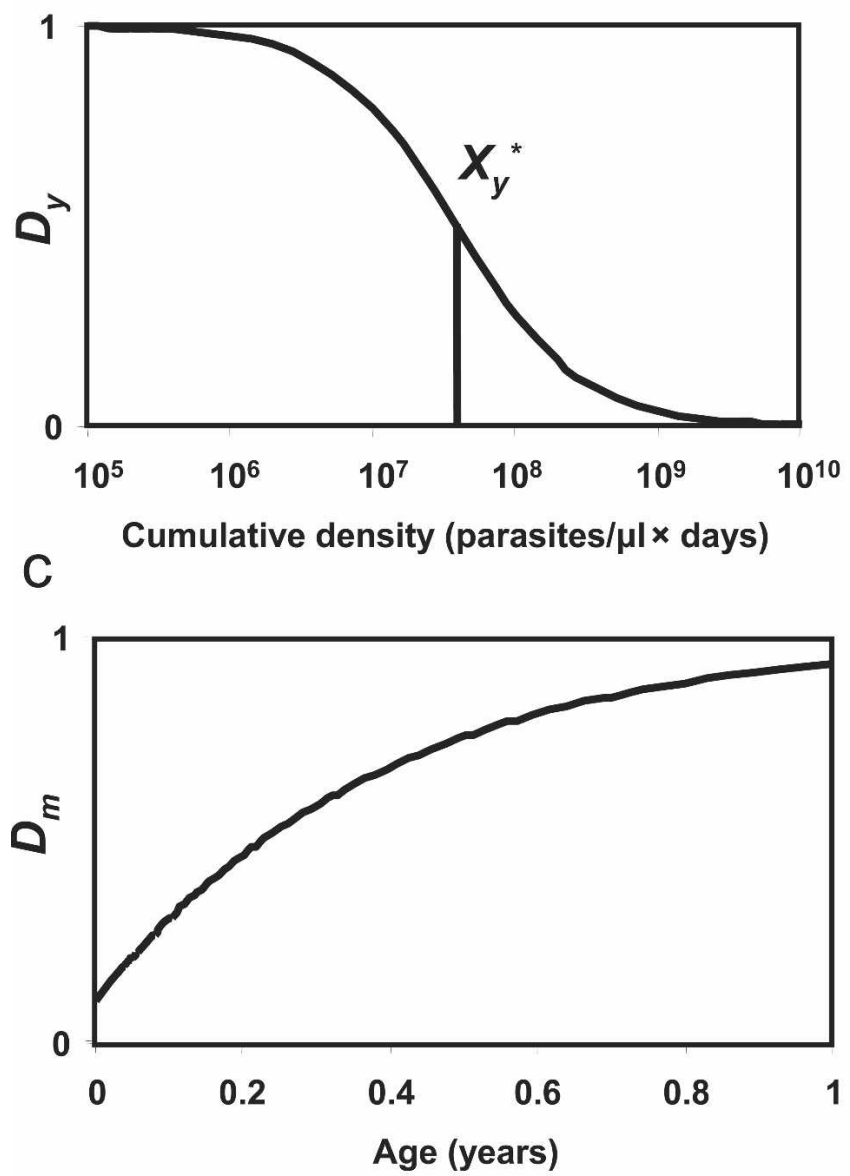

b
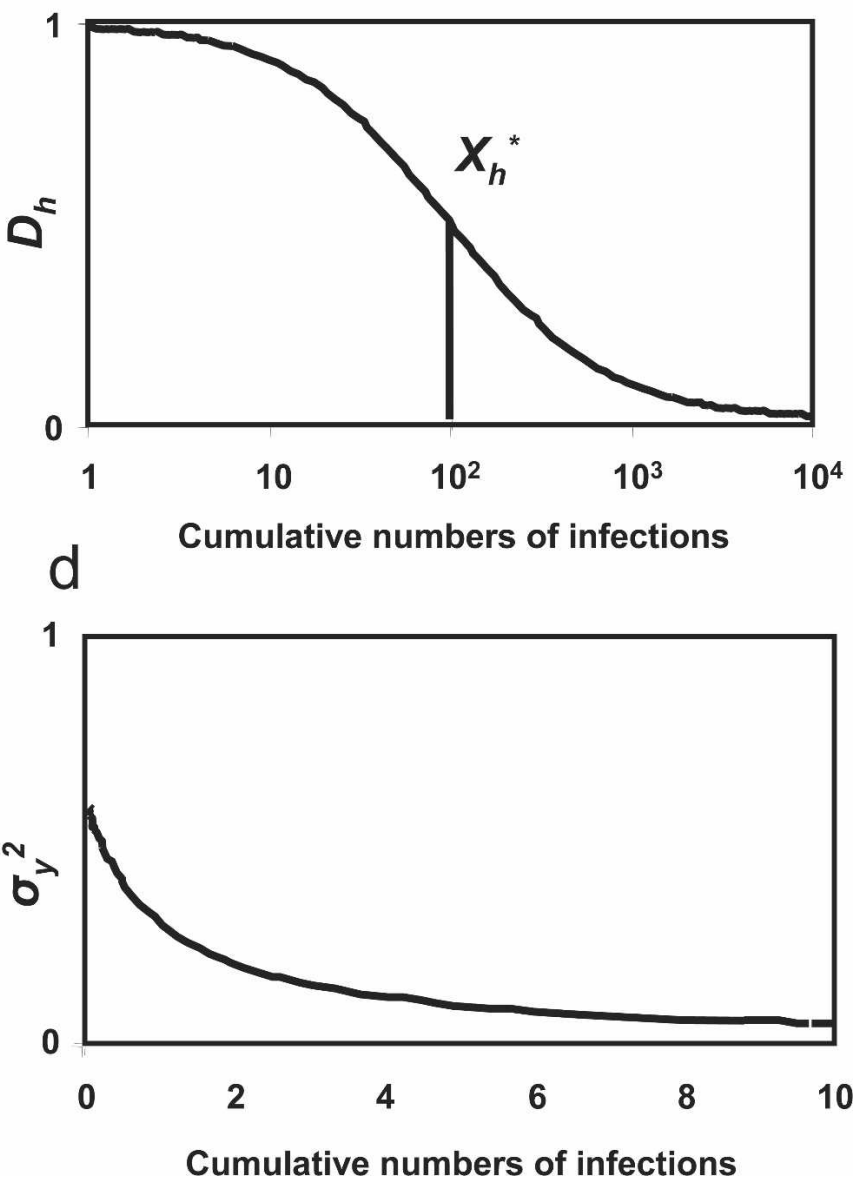

FIGURE 3. Effects of acquired immunity on parasite density. a, Cumulative density of asexual parasitemia. Relationship between the cumulative density of parasites experienced and the trigger $D_{y}$ (equation 4). b. Cumulative number of infections. Relationship between the cumulative number of infections and $D_{h}$ (equation 6). c, Age of the host. Age-dependent decay of maternal immunity (equation 7). d, Within-host variation of parasite densities. Variation around expected parasite densities within individual hosts as function of cumulative parasite densities experienced (equation 10). 
by least squares fitting of local quadratic approximations to the (stochastic) log likelihood surface.

Validation of model for age-prevalence. In addition to fitting the model to data we compared the predicted relationship between prevalence in children less than five years of age and EIR with the meta-analysis of Beier and others. ${ }^{28}$

\section{RESULTS}

We optimized our model for the control of asexual blood stages of $P$. falciparum in terms of the biologic plausibility of the mechanisms for the control of parasites, the agreement with gross features of the actual field data, and the fit to six datasets across a range of transmission intensities as measured by the joint log likelihood. The final model that we propose incorporates most of the processes that we initially envisaged. In particular, it includes stochastic variation in the duration of infections, and in the densities that they achieve at each time point. These densities decrease in general as a sigmoidal function of the cumulative density experienced by the host (equation 4) (Figure 3a), with the critical value of the curve $X_{y}^{*}$, which correspond to a mean age of 7.1 years $(\mathrm{SD}=$ 10.1) in the lowest EIR setting (Rafin Marke) and 5.3 years $(\mathrm{SD}=8.5)$ in Navrongo.
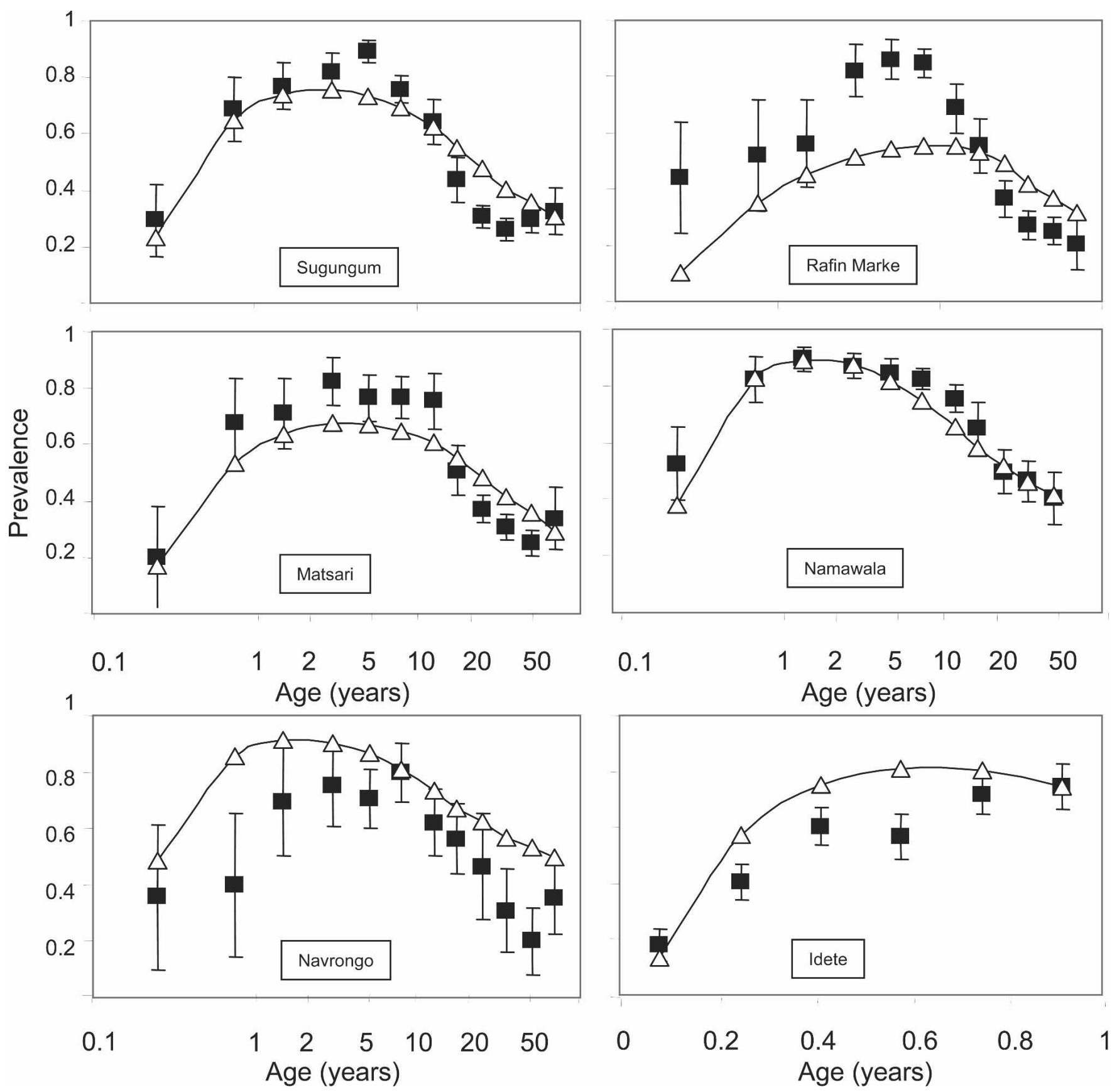

FIGURE 4. Comparison of predicted and observed age-prevalence curves. $\boldsymbol{\Xi}=$ data (error bars show $95 \%$ confidence intervals); $\Delta=$ model predictions. 
The densities also decrease with the cumulative numbers of infections experienced (equation 6) (Figure 3b) with the critical value of this curve $X_{h}^{*}$, which corresponded to a mean age of 27.5 years $(\mathrm{SD}=2.5)$ in Rafin Marke and 11.4 years $(\mathrm{SD}=1.2)$ in Navrongo. The predicted age pattern of the effects of maternal immunity on the densities (equation 7) (Figure 3c) shows the expected decay of this component of immunity in the first few months of life. The distributions of the densities vary by individual host (equation 2), and there is also stochastic within-host variation in the densities by exposure (equation 10) (Figure 3d).

The model did not include any explicit effect of parasite density on the infections emerging from the liver (see Smith and others ${ }^{16}$ ), and the best fitting value of $D_{x}$, explicitly quantifying interactions between co-infections (equation 9), was 0 , which limited the interactions between concurrent infections to those induced by acquired immunity. We explored several different parameterizations to try to capture interactions between co-infections, but we were not able to improve the fit of our models.

The model also assumed that each inoculation behaves as a single parasite clone. This is a simplification since many oocysts in the field are heterozygous ${ }^{29,30}$ and result in mixed populations of sporozoites (Ranford-Cartwright L, unpublished data). However, we were not able to improve the fit of the model by allowing for diversity in the inoculum.

A good fit of the model to the parasite densities could only be achieved by including parameters to allow for biases introduced by different methods for quantifying parasite densities. We aimed to adjust all the modeled densities to the same scale as that in the malariatherapy studies by introducing two parameters $v$, one for the Garki dataset (where the nominal densities were much lower than our model predicted), and one for the other field studies, where the nominal densities were higher than our predictions (Table 3). These adjustments ensured that our predicted densities in naive individuals corresponded on average to those in malariatherapy patients but do not allow us to separate ethnic variations from effects of differences among study sites in the quantification of parasitemia. In all settings, $P$. falciparum showed the characteristic age-prevalence pattern found in malaria-endemic areas, with the highest prevalence in young children, reaching almost $100 \%$ in those sites with EIRs > 200 infectious bites per person per year (Figure 4).

The age of peak prevalence was younger in the highest transmission sites and moves to older ages in lower transmission settings, but there is little difference between sites in the prevalence in older children and adults. These patterns were well reproduced by our model, with clear separation between the sites in the predicted age-prevalence curves in young children at all except the highest transmission rates, with peak prevalence at the age of one year in the highest transmission sites and in adolescents in the lowest transmission site of Rafin Marke.

Despite the highest recorded EIR in Navrongo, the prevalence of $P$. falciparum was relatively low in the young children; thus, this setting had an anomalously high age of peak prevalence (Figure 4). ${ }^{31}$ The age at which the multiplicity reaches a peak is also anomalously high in Navrongo, in comparison to other sites that have been studied (Figure 5). ${ }^{13,31}$

The highest geometric mean parasite densities rather than the peaks in prevalence were found in younger children, with

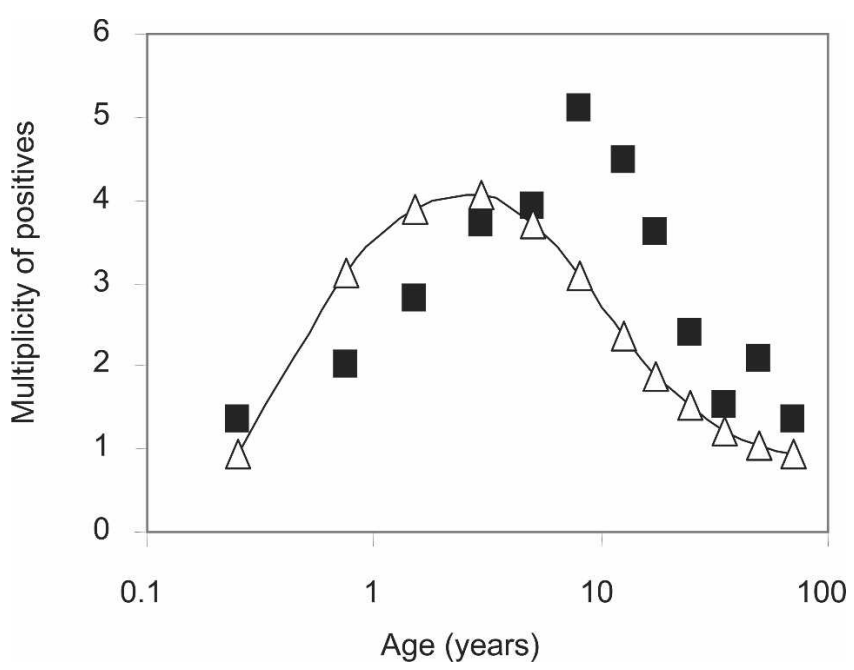

FIGURE 5. Comparison of predicted versus observed multiplicity of infection. $=$ data for Navrongo; $\Delta=$ model predictions.

gradual decreases with age in the geometric mean densities among the infected individuals (Figure 6). There was little peak shift in the geometric mean densities in either the data or the model predictions, which suggested that the highest densities should be found in the 1-2-year-old children. Comparison of the model predictions with the summary of field studies of prevalence in children less than five years found a good fit, with only a small tendency for the predicted prevalence to be below that observed (Figure 7).

Since the model was fitted only to cross-sectional data, it was not expected to give good predictions of longitudinal patterns within individual hosts. Comparison of individual parasitologic profiles with the patterns observed in malaria therapy patients confirmed that within host variation was less than that observed in actual infections of naive hosts.

\section{DISCUSSION}

The model we present for the processes involved in controlling the asexual blood stages of $P$. falciparum infections provides a foundation for estimating the likely impact of variations in malaria transmission and of control of parasite densities on the parasitologic burden in endemic areas. It forms a central component of a comprehensive model to assess and quantify the epidemiologic and economic effects of introducing malaria vaccines. ${ }^{32}$ In addition to these applications, our analyses of models for the control of asexual blood stages can also be used to test hypotheses about the nature of acquired immunity and malaria transmission dynamics.

The realism of the model presented here is limited by a number of gaps in our current knowledge of malaria. For example, no attempt has been made to simulate detailed immunologic processes. This is justified by the lack of agreed immunologic proxy markers for protection in malaria. Thus, models of malaria immunity that aim to predict morbidity and mortality cannot be validated against immunologic data. Modeling T cell dynamics or antibody levels would thus serve only to increase the complexity of the models without adding to the validity. Our model assumed that the main mechanisms controlling parasite densities do not decay in the absence of 

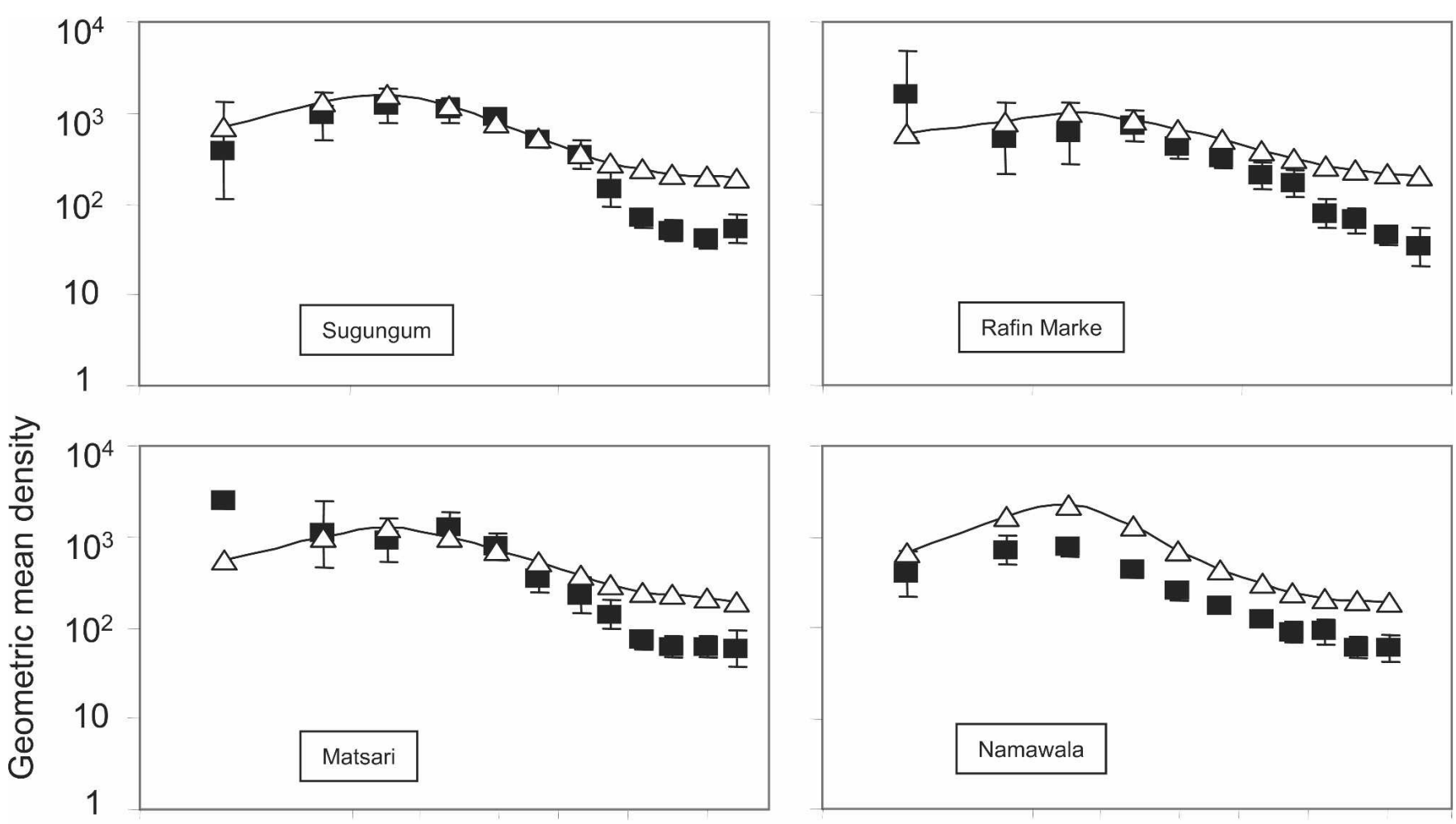

\section{$\begin{array}{lllllll}0.1 & 1 & 2 & 5 & 10 & 20 & 50\end{array}$ Age (years)}
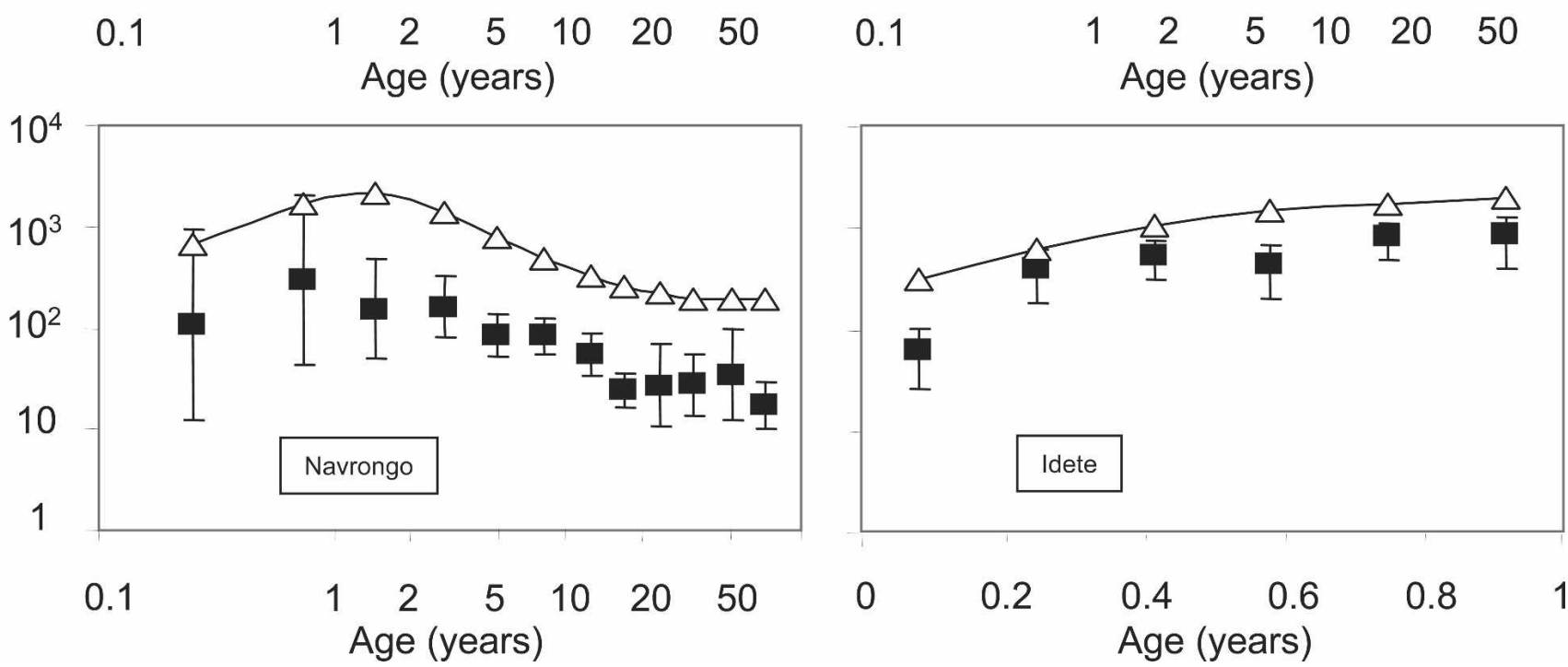

FIGURE 6. Comparison of predicted versus observed age-density curves $\boldsymbol{\|}=$ data (error bars show $95 \%$ confidence intervals); $\Delta=$ model predictions.

stimulation because we have no comprehensive database from which to estimate rates of decay. It would be possible to analyze predictions from sets of models fitted to the same data, assuming different rates of decay, but this would require substantially more computing power than the analysis of a single model.

Inhabitants of endemic areas who temporarily move away are more vulnerable to malaria when they return, but it is unclear whether this results from loss of anti-parasite immunity or of parasitologic tolerance. Some specific immune responses against $P$. falciparum have been shown to be highly labile (e.g., Kinyanjui and others ${ }^{33}$ ), but the epidemiologic consequences of this are yet to be elucidated. Even after sev- eral decades free of malaria, people in the central highlands of Madagascar retained some protective immunity to $P$. falciparum. $^{34}$

To reproduce realistic age-prevalence and age-density curves, we required only to simulate effects of acquired immunity on parasite densities, without any decay in immunity over time or any explicit effect on duration of infection. In common with many other diseases with acquired immunity, ${ }^{35,36}$ as $P$. falciparum transmission increases, there is a characteristic decrease in the age at which the maximum prevalence is reached and increases in the actual peak prevalence. Our model was also able to reproduce these shifts.

Terms both in the cumulative parasite load, $X_{y}$, and cumu- 


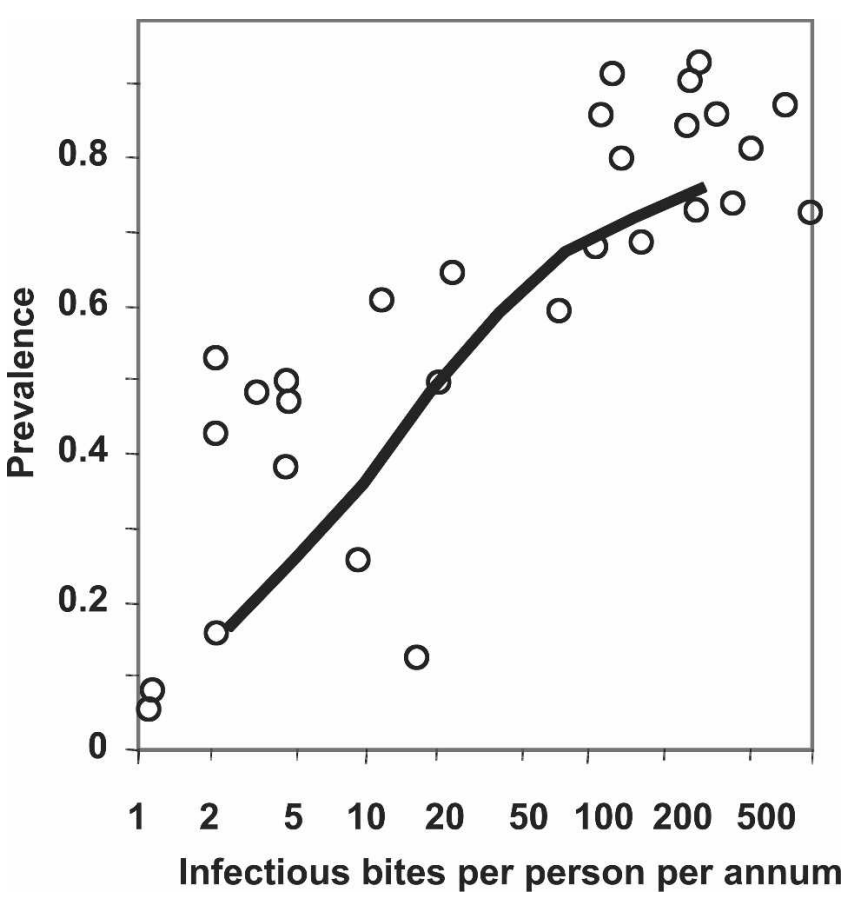

FIGURE 7. Comparison of model predictions with observed prevalence in children less than five years of age in 31 sites across Africa. $\bigcirc=$ field data reported by Beier and others. ${ }^{28}$ The line corresponds to predictions of our model made using the seasonal pattern of inoculations in Namawala scaled to different values of the annual entomologic inoculation rate.

lative number of infections, $X_{h}$, were needed, which confirmed that models are inadequate in which acquired immunity is a simple function only of the number of strains to which the host has been exposed. The quantitative load of parasites resulting from repeated inoculations is also important in our proposed model. This could reflect effects of the repertoire of antigen variants that have been expressed in the host, as well as undifferentiated effects of the actual number of parasites. Our formulation differs from models that assume the parasite population to comprise a limited number of strains, or that strain-specific immunity can lead to complete protection.

As with all mathematical models for infectious diseases, we made a number of simplifications. First, we assumed that ethnic differences in the response to $P$. falciparum malaria between sites are small, although such differences are known to occur in sub-Saharan Africa. ${ }^{37,38}$

Although our model showed satisfactory overall fits to the various datasets across a wide range of transmission intensities, a somewhat poorer fit was observed with the Navrongo data. A likely explanation for this relatively poor fit to this more recently collected dataset is that we assumed that the impact of anti-malaria treatment was negligible. This assumption is clearly appropriate in the case of the historical data from sites where there was little or no effective treatment, and enabled us to avoid the need to model treatment effects. However, in sub-Saharan Africa early diagnosis and treatment is currently the backbone of malaria control. ${ }^{39} \mathrm{We}$ needed to use recent data to fit the model to age-multiplicity patterns because genotyping techniques have only recently become available. The data from Navrongo that we used for this come from an area with generally poor access to pri- mary health services, although there are four health centers and a hospital. A fraction of the population sleeps under mosquito nets after a successful trial carried out a decade ago, ${ }^{40}$ and a pilot community health program has treated many of the acute illnesses, including fever episodes, in young children. ${ }^{41}$ The poorer fit of our model to the Navrongo data than to the other datasets suggests that these interventions have reduced levels of malaria infection in Navrongo below those to be expected at the high inoculation rates observed there.

Other limitations arise because our model was fitted to cross-sectional data, rather than to patterns of variation in parasitemia within individuals, and therefore it is more successful in reproducing the former than the latter. Longitudinal patterns within individuals might be better reproduced by fitting within-host models explicitly incorporating effects of antigenic variation to repeated assessments of parasitemia with short time intervals. Such models have been fitted to data from malariatherapy patients ${ }^{3,4,42}$ but there is no agreement that any particular such model is appropriate. By basing the time course of parasitemia on an empirical description of the malariatherapy data, we attempted to incorporate the dynamics of antigenic variation without explicitly modeling the processes that lead to it. Because our model is individual based and therefore makes predictions of sequential patterns of parasitemia within simulated hosts, it would be possible to amend it to include a more realistic model of within-host dynamics but there are few datasets from endemic areas to which longitudinal patterns of parasitemia with short timeintervals can be fitted.

We conclude that this model appears to reproduce reasonably well the parasitologic patterns seen in malariologic surveys in endemic areas. Development of improved models will require fitting to data on longitudinal patterns of parasitemia in semi-immune individuals, in particular to data from people whose exposure is interrupted by well-documented periods of protection from infection. Such models are needed to understand the consequences of malaria interventions in areas of infrequent or unstable transmission. The present model is appropriate for predicting the impact of interventions such as vaccination in the context of continual exposure to $P$. falciparum.

Received September 18, 2005. Accepted for publication February 7, 2006.

Acknowledgments: We thank Dan Anderegg for editorial assistance and Paulette Rosé for assistance in retrieving the Garki data. We also thank the members of the Technical Advisory Group (Michael Alpers, Paul Coleman, David Evans, Brian Greenwood, Carol Levin, Kevin Marsh, F. Ellis McKenzie, Mark Miller, and Brian Sharp), the Project Management Team at the Program for Appropriate Technology in Health (PATH) Malaria Vaccine Initiative, and GlaxoSmithKline Biologicals S.A for their assistance.

Financial support: The mathematical modeling study was supported by the PATH Malaria Vaccine Initiative and GlaxoSmithKline Biologicals S.A.

Disclaimer: Publication of this report and the contents hereof do not necessarily reflect the endorsement, opinion, or viewpoints of the PATH Malaria Vaccine Initiative or GlaxoSmithKline Biologicals S.A.

Authors' addresses: Nicolas Maire, Thomas Smith, and Amanda Ross, Swiss Tropical Institute, Socinstrasse 57, PO Box, CH-4002, Basel, Switzerland, Telephone: 41-61-284-8273, Fax: 41-61-284-8105, 
E-mails: nicolas.maire@unibas.ch,Thomas-A.Smith@unibas.ch, and amanda.ross@unibas.ch. Seth Owusu-Agyei, Navrongo Health Research Center, Ministry of Health, Navrongo Upper East Region, Ghana. Klaus Dietz, Department of Medical Biometry, University of Tübingen, Westbahnhofstrasse 55, 72070 Tübingen, Germany, Telephone: 49-7071-29-78253, Fax: 49-7071-29-5075. E-mail: klaus .dietz@uni-tuebingen.de. Louis Molineaux, Peney-Dessus, CH-1242 Satigny, Geneva, Switzerland.

Reprint requests: Thomas Smith, Swiss Tropical Institute, Socinstrasse 57, PO Box, CH-4002, Basel, Switzerland. E-mail: ThomasA.Smith@unibas.ch.

\section{REFERENCES}

1. Molineaux L, Muir DA, Spencer HC, Wernsdorfer WH, 1988 The epidemiology of malaria and its measurement. Wernsdorfer WH, Mc Gregor I, eds. Malaria, Principles and Practice of Malariology. Edinburgh: Churchill Livingstone, 999-1089.

2. Alonso PL, Sacarlal J, Aponte J, Leach A, Macete E, Milman J, Mandomando I, Spiessens B, Guinovart C, Espasa M, Bassat $\mathrm{Q}$, Aide P, Ofori-Anyinam O, Navia MM, Corachan S, Ceuppens M, Dubois MC, Demoitie MA, Dubovsky F, Menendez C, Tornieporth N, Ballou WR, Thompson R, Cohen J, 2004. Efficacy of the RTS,S/AS02A vaccine against Plasmodium falciparum infection and disease in young African children: randomised controlled trial. Lancet 364: 1411-1420.

3. Molineaux L, Diebner HH, Eichner M, Collins WE, Jeffery GM, Dietz K, 2001. Plasmodium falciparum parasitaemia described by a new mathematical model. Parasitology 122: 379-391.

4. Paget-McNicol S, Gatton M, Hastings I, Saul A, 2002. The Plasmodium falciparum var gene switching rate, switching mechanism and patterns of parasite recrudescence described by mathematical modelling. Parasitology 124: 225-235.

5. Dietz K, Molineaux L, Thomas A, 1974. A malaria model tested in the African savannah. Bull World Health Organ 50:347-357.

6. Aron JL, 1988. Mathematical-modeling of immunity to malaria. Math Biosci 90: 385-396.

7. Struchiner CJ, Halloran ME, Spielman A, 1989. Modeling malaria vaccines. I: New uses for old ideas. Math Biosci 94: 87-113.

8. Elderkin RH, Berkowitz DP, Farris FA, Gunn CF, Hickernell FJ, Kass SN, Mansfield FI, Taranto RG, 1977. On the steady state of an age-dependent model for malaria. Lakschmikantham V, ed. Nonlinear Systems and Applications. New York: Academic Press, 491-512.

9. Molineaux L, Gramiccia G, 1980. The Garki Project. Geneva: World Health Organization.

10. Macdonald G, 1957. The Epidemiology and Control of Malaria. London: Oxford, United Kingdom: Oxford University Press.

11. Sama W, Killeen G, Smith T, 2004. Estimating the duration of Plasmodium falciparum infection from trials of indoor residual spraying. Am J Trop Med Hyg 70: 625-634.

12. Gupta S, Day KP, 1994. A theoretical framework for the immunoepidemiology of Plasmodium falciparum malaria. Parasite Immunol 16: 361-370.

13. Smith T, Beck H-P, Kitua A, Mwankusye S, Felger I, Fraser-Hurt N, Irion A, Alonso PL, Teuscher T, Tanner M, 1999. Age dependence of the multiplicity of Plasmodium falciparum infections and of other malariological indices in an area of high endemicity. Trans R Soc Trop Med Hyg 93 (Suppl 1): 15-20.

14. Smith T, Vounatsou P, 2003. Estimation of infection and recovery rates for highly polymorphic parasites when detectability is imperfect, using hidden Markov models. Stat Med 22: 17091724.

15. Collins WE, Jeffery GM, 1999. A retrospective examination of sporozoite- and trophozoite-induced infections with Plasmodium falciparum in patients previously infected with heterologous species of Plasmodium: effect on development of parasitologic and clinical immunity. Am J Trop Med Hyg 61: 36-43.

16. Smith T, Maire N, Dietz K, Killeen GF, Vounatsou P, Molineaux L, Tanner M, 2006. Relationships between the entomologic inoculation rate and the force of infection for Plasmodium falciparum malaria. Am J Trop Med Hyg 75 (Suppl 2) 11-18.

17. Kitua A, Smith T, Alonso PL, Masanja H, Urassa H, Menendez C, Kimario J, Tanner M, 1996. Plasmodium falciparum malaria in the first year of life in an area of intense and perennial transmission. Trop Med Int Health 1: 475-484.

18. Vounatsou P, Smith T, Kitua A, Alonso PL, Tanner M, 2000. Apparent tolerance of Plasmodium falciparum in infants in a highly endemic area. Parasitology 120: 1-9.

19. Shute GT, 1988. The microscopic diagnosis of malaria. Wernsdorfer WH, Mc Gregor I, eds. Malaria, Principles and Practice of Malariology. Edinburgh: Churchill Livingstone, 781-784.

20. Earle WC, Perez M, 1932. Enumeration of parasites in the blood of malarial patients. J Lab Clin Med 17: 1124-1132.

21. Glynn JR, Bradley DJ, 1995. Inoculum size, incubation period and severity of malaria. Analysis of data from malaria therapy records. Parasitology 110: 7-19.

22. Ross A, Smith T, 2006. The effect of malaria transmission intensity on neonatal mortality in endemic areas. Am J Trop Med Hyg 75 (Suppl 2): 74-81.

23. Charlwood JD, Smith T, Lyimo E, Kitua A, Masanja H, Booth M, Alonso PL, Tanner M, 1998. Incidence of Plasmodium falciparum infection in infants in relation to exposure to sporozoite-infected anophelines. Am J Trop Med Hyg 59: 243-251.

24. Smith T, Charlwood JD, Kihonda J, Mwankusye S, Billingsley P, Meuwissen J, Lyimo E, Takken W, Teuscher T, Tanner M, 1993. Absence of seasonal variation in malaria parasitaemia in an area of intense seasonal transmission. Acta Trop 54: 55-72.

25. Beier JC, Oster CN, Onyango FK, Bales JD, Sherwood JA, Perkins PV, Chumo DK, Koech DV, Whitmire RE, Roberts CR, 1994. Plasmodium falciparum incidence relative to entomologic inoculation rates at a site proposed for testing malaria vaccines in western Kenya. Am J Trop Med Hyg 50: 529-536.

26. Kirkpatrick S, Gelatt CD Jr, Vecchi MP, 1983. Optimization by simulated annealing. Science 220: 671-680.

27. Press WH, Flannery BP, Teukolsky SA, Vetterling WT, 1988. Numerical Recipes in C: the Art of Scientific Computing. Cambridge, United Kingdom: Cambridge University Press.

28. Beier JC, Killeen G, Githure JI, 1999. Short report: entomologic inoculation rates and Plasmodium falciparum malaria prevalence in Africa. Am J Trop Med Hyg 61: 109-113.

29. Babiker H, Ranford-Cartwright L, Currie D, Charlwood JD, Billingsley P, Teuscher T, Walliker D, 1994. Random mating in a natural population of the malaria parasite Plasmodium falciparum. Parasitology 109: 413-421.

30. Paul RE, Packer MJ, Walmsley M, Lagog M, Ranford-Cartwright L, Paru R, Day KP, 1995. Mating patterns in malaria parasite populations of Papua New Guinea. Science 269: 1709-1711.

31. Owusu-Agyei S, Smith T, Beck H-P, Amenga-Etego L, Felger I, 2002. Molecular epidemiology of Plasmodium falciparum infections among asymptomatic inhabitants of a holoendemic malarious area in northern Ghana. Trop Med Int Health 7: 421-428.

32. Smith T, Killeen G, Maire N, Ross A, Molineaux L, Tediosi F, Hutton G, Utzinger J, Dietz K, Tanner M, 2006. Mathematical modeling of the impact of malaria vaccines on the clinical epidemiology and natural history of Plasmodium falciparum malaria. Am J Trop Med Hyg 75 (Suppl 2): 1-10.

33. Kinyanjui SM, Bull P, Newbold C, Marsh K, 2003. Kinetics of antibody responses to Plasmodium falciparum-infected erythrocyte variant surface antigens. J Infect Dis 187: 667-674.

34. Deloron P, Chougnet C, 1992. Is immunity to malaria really short-lived? Parasitol Today 8: 375-378.

35. Anderson RM, May RM, 1991. Infectious Diseases of Humans: Dynamics and Control. Oxford, United Kingdom: Oxford University Press.

36. Woolhouse ME, 1998. Patterns in parasite epidemiology: the peak shift. Parasitol Today 14: 428-434.

37. Greenwood BM, Groenendaal F, Bradley AK, Greenwood AM, Shenton F, Tulloch S, Hayes R, 1987. Ethnic differences in the prevalence of splenomegaly and malaria in The Gambia. Ann Trop Med Parasitol 81: 345-354.

38. Modiano D, Petrarca V, Sirima BS, Nebie I, Diallo D, Esposito F, Coluzzi M, 1996. Different response to Plasmodium falciparum malaria in west African sympatric ethnic groups. Proc Natl Acad Sci U S A 93: 13206-13211.

39. World Health Organization, 1993. A Global Strategy for Malaria Control. Geneva, World Health Organization.

40. Binka F, Kubaje A, Adjuik M, Williams LA, Lengeler C, Maude 
G, Armah GE, Kajihara B, Adiamah JH, Smith PG, 1996. Impact of permethrin impregnated bednets on child mortality in Kassena-Nankana district, Ghana: a randomized controlled trial. Trop Med Int Health 1: 147-154.

41. Binka F, Nazzar A, Phillips JF, 1995. The Navrongo Community Health and Family Planning Project. Stud Fam Plann 26: 121139.

42. Recker M, Nee S, Bull P, Kinyanjui S, Marsh K, Newbold C, Gupta S, 2004. Transient cross-reactive immune responses can orchestrate antigenic variation in malaria. Nature 429: 555-558.

43. United Nations, 2002. Demographic Yearbook 2000. New York: United Nations.

44. INDEPTH Network, 2002. Population, Health and Survival at INDEPTH Sites. Ottawa, Ontario, Canada: International Development Research Centre.

45. Alonso PL, Smith T, Schellenberg JR, Masanja H, Mwankusye S, Urassa H, Bastos de Azevedo I, Chongela J, Kobero S, Menendez C, 1994. Randomised trial of efficacy of SPf66 vaccine against Plasmodium falciparum malaria in children in southern Tanzania. Lancet 344: 1175-1181.

\section{APPENDIX \\ DEMOGRAPHIC STRUCTURE OF THE SIMULATED POPULATION}

The simulation requires a demographic model for the human population in which the following are needed.

1) Population size is held constant (this ensures that the computational effort and denominators remain similar throughout the simulation. In some applications we do not know at the outset how much time we must simulate; we do not want the simulated population to be continuously growing).

2) The age distribution remains approximately constant, so that models for parasite dynamics can reach equilibrium (assuming constant host/vector ratios). The infectious reservoir depends on the age distribution of the human population so a stable age distribution is required for parasitologic equilibrium.

3) The age distribution reflects that of actual contemporary populations to ensure correct overall measures of disease burden and transmissibility.
Real populations are created by processes that vary over time, and in sub-Saharan Africa they are growing. ${ }^{43}$ In a simulated population it is not in general possible to keep the age distribution stable by applying current birth and death rates. Usually a stable age distribution can only be maintained by removing more simulated individuals at each age and time point than the deaths predicted by a realistic mortality model. We separate processes maintaining the age-distribution of the human population from the demographic effects of malaria, which we do not aim to model, by simulating out migration. We use age-specific out-migration rates to those needed to maintain a stable population. In fitting our parasitologic model we do not simulate any mortality. The same approach is used in models that do simulate mortality, in which the out migration rates are reduced to compensate for the deaths.

Age distribution of the simulated human population. We simulate age distributions based on those recorded for African demographic surveillance sites. ${ }^{44}$ Stable life tables cannot be constructed for these age distributions without including age-specific immigration. We avoid simulating immigration because this would require us to clone simulated individuals, which would alter the variance structure. We fit a continuous parametric curve to smooth the age distribution because the distributions summarized in published data are themselves smooth, and fewer in-migrations are needed. A bathtub function for mortality rates (higher in infants and older people) gives a good fit to historical European data (Safan M, unpublished data) and separates parameters of the age distribution from those of population growth.

If $\rho$ is the Malthusian parameter, corresponding to the exponent of an exponential growth model, then a stationary age-distribution is given by $f(a)$ where

$$
f(a)=\frac{e^{-\rho a-M(a)}}{\int_{0}^{\infty} e^{-\rho a-M(a)} d a} \text { and M(a) } \mu_{0} \frac{1-e^{-\alpha_{0} a}}{\alpha_{0}}+\mu_{1} \frac{e^{\alpha_{1} a}-1}{\alpha_{1}}
$$

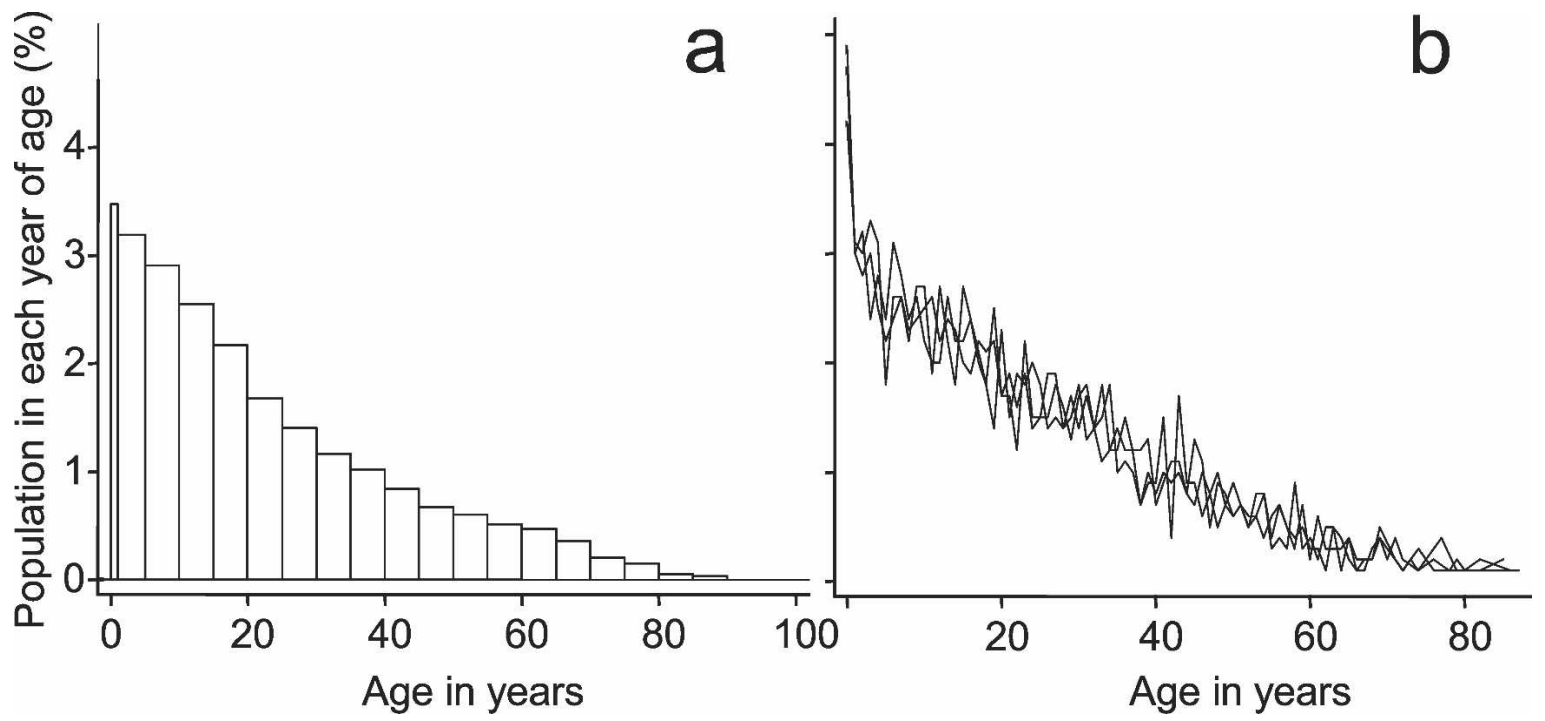

FIGURE 8. Age distributions of the population a, Field data from Ifakara, Tanzania. ${ }^{44} \mathbf{b}$, Simulated population distributions of 1,000 individuals at 3 cross-sectional time points $(\mathrm{t}=10,000,50,000$, and 100,000). 
Fitting the parameters of the age distribution. We set $\rho$, the population growth rate, to 0 and estimate the parameters $\mu 0, \mu 1, \alpha 0$, and $\alpha 1$ and by least squares fitting of the logarithmically transformed percentages of the population in each age group to those of real African populations. Because the observed age distribution is aggregated into a limited number of groups, the parameters $\mu 0$ and $\alpha 0$ are difficult to fit. We require a high number of removals in the first year of life, so models with high infant mortality do not require immigration of infants. We specify a high removal rate of in- fants and constrain the age-specific removal rates in the first year of life so that there are four neonatal removals for every six post-neonatal one. The age-specific removal rates of the simulated population are then $\mu(\mathrm{a})=$ $\mu_{0} e^{-\alpha o a}+\mu 1 \mathrm{e}^{\alpha 1 a}$.

At each five-day time point, we compare the age-specific cumulative numbers of individuals to that of the target population, and excess individuals are out migrated. After a run-in period, the age distribution based on Tanzanian data (Figure $8 \mathrm{a}$ ) is approximately stable (Figure $8 \mathrm{~b}$ ). 